\title{
Deep Learning Approach based on 1D Convolutional Neural Networks for predicting Sudden Cardiac Arrest
}

\author{
viswavardhanreddy karna ${ }^{1}$ and Navin Kumar $^{2}$ \\ ${ }^{1}$ Jain University \\ ${ }^{2}$ Amrita Vishwa Vidyapeetham Amrita School of Engineering Bengaluru
}

July 2, 2021

\begin{abstract}
Sudden cardiac arrest (SCA) is a serious heart problem which occur without any apparent symptoms and warning. SCA leads to a large amount of fatality. Hence, predicting the occurrence of SCA is very important. Current traditional methods for predicting a ventricular fibrillation ( $\mathrm{VF}$ ) onset requires a considerably longer-monitoring time of patients, resulting in unprecedented complications. The emerging technology, in particular machine learning techniques have gained popularity due to the effectiveness. However, most of existing techniques heavily rely on a set of hand-engineered features which may results in poor generalization when presented out of distribution patient data. On the other hand, existing deep learning approaches rely heavily on large training data which is not available publicly. In this study, we proposed a deep learning approach based on 1D convolutional neural network trained with discrete Fourier transform (DFT) features from raw electrocardiogram (ECG) signals. As demonstrated in the results, our method can predict accurately around 90 minutes before the onset of SCA with an accuracy of $96 \%$. The advance prediction might save many lives. That is, optimized deep learning models offer better generalization than hand-crafted features while detecting long-term signal relations.
\end{abstract}

\section{Hosted file}

Final_SCD_Deep_wiley.docx available at https://authorea.com/users/359086/articles/528781deep-learning-approach-based-on-1d-convolutional-neural-networks-for-predicting-suddencardiac-arrest 